\title{
The relationship between p53, Bcl2 proteins and histological grade in colorectal adenocarcinoma associated with type 2 diabetes and obesity
}

\author{
Popescu-Vâlceanu Horaţiu-Cristian ${ }^{1}$, Stoicea Mihai ${ }^{2}$, Enache Valentin ${ }^{3}$, Mustatea Petronel ${ }^{4}$, \\ Rusu Emilia ${ }^{4}$, Drăgut Ramona ${ }^{4}$ and Radulian Gabriela ${ }^{4,5}$ \\ ${ }^{1}$ Carol Davila" University of Medicine and Pharmacy, Bucharest, Romania, \\ ${ }^{2}$ Central Lab Synevo, Bucharest, Romania, \\ ${ }^{3}$ Emergency Clinical Hospital- Pathology Departament, Bucharest, Romania, \\ ${ }^{4}$ Carol Davila University of Medicine and Pharmacy, Bucharest, Romania and \\ ${ }^{5}$ National Institute of Diabetes, Nutrition and Metabolic Diseases "Prof. N. C. Paulescu", Bucharest, Romania
}

\begin{abstract}
Introduction: The aim of our study was to evaluate the expression of p53 protein in colorectal adenocarcinoma in diabetic (type 2) obese vs non-obese vs and to analyse p53, Bcl2 proteins expression in type 2 diabetic vs non-diabetic patients based on histological grade.
\end{abstract}

Material and Methods: In a retrospective study we analyzed all hospitalized patients with colorectal cancer between 2011-2015 in Bucharest Emergency Hospital, according to International Statistical Classification of Diseases classification and Related Health Problems 10th Revision.

The p53 and Bcl2 proteins expressions were investigated by automated immunohistochemistry BenchMark XT Ventana platform using dual Bcl2-p53 protocol in the Histopathology Department of the Central Reference Laboratory Synevo.

Results: We identified in order of appearance only the cases with appropriate inclusion criteria, 95 cases of colorectal adenocarcinoma: 52 were type 2 diabetic patients (33males /19 females, mean age 70.2 years) and 43 non-diabetics ( 30 males /13 females mean age 69.5 years). There were $15 \mathrm{vs} 2$ obese subjects in diabetic/non-diabetic patients compared to $37 \mathrm{vs} 41$ with normal weight.

Our data showed that obese diabetic patients associate more frequently the overexpression of p53 protein in colorectal adenocarcinoma in the $80 \%$ (12/15 of cases) comparative with non-obese diabetic patients $40.5 \%$ (15/37 cases) or non-diabetics and non-obese controls $36.6 \%(15 / 41$ cases $\mathrm{p}=0.024)$.

Regarding histological grade, diabetic patients associated low-grade colorectal tumors (78.8\% of cases) compared with nondiabetics (58.1\% of cases) and non-diabetic patients associated high-grade colorectal tumors increased (41.9\%) compared to diabetics $(21.2 \%)$, with statistical significance $(\mathrm{p}=0.043)$.

Diabetics compared with non-diabetics associated an oversexpression of all immunophenotypes in the low histological grade colorectal adenocarcinoma: Bcl2-/p53- immunophenotype in $62 \%$ vs $53 \%$ of cases $(\mathrm{p}=0.836)$; for $\mathrm{Bcl} 2+/ \mathrm{p} 53$ - immunophenotype in $67 \%$ vs $43 \%$ of cases $(\mathrm{p}=0.064)$; for Bcl2-/p53 + immunophenotype in $77 \%$ vs $71 \%$ of cases $(\mathrm{p}=0.489)$ and for Bcl $2+/ \mathrm{p} 53+\mathrm{immunophe}-$ notype in $100 \%$ vs $33 \%$ of cases $(p=0.333)$.

Discussion: It is known that the protein p53 is a powerful transcriptor factor acting as checkpoint controlling the differentiation of the various cells including adipocytes and also possible enterocytes (explaining the higher frequency of colorectal cancer which has been associated with the increased proliferation of adipocytes characterizing the obesity).Due to the protection conferred by a normal p53 protein its upregulation could be a new target for the treatment of obesity. Interesting our work revealed that diabetic patients associated low-grade colorectal adenocarcinoma with an oversexpression of all immunophenotypes.

\section{Conflict of Interest}

There is no conflict of interest 